\title{
Modeling Acoustic Signal Fluctuations Induced by Sea Surface Roughness
}

\author{
Robert M. Heitsenrether, Mohsen Badiey \\ Ocean Acoustics Laboratory, College of Marine Studies, University of Delaware, Newark, DE 19716
}

\begin{abstract}
An empirical fetch-limited ocean wave spectrum has been combined with an acoustic ray-based model to predict the acoustic signal time-angle fluctuations induced by sea surface roughness. Rough sea surface realizations are generated and used as sea surface boundaries with the acoustic model. To validate this model, results are compared against experimental data collected in a fetch limited region. These data includes simultaneous wind speed and acoustic propagation (1-18 kHz) measurements in a fetch limited coastal region. Modeled time-angle fluctuations compare well with field data at lower wind speeds $(<10 \mathrm{~m} / \mathrm{s})$.
\end{abstract}

\section{INTRODUCTION}

Surface waves are among several environmental parameters that can have significant influence on the propagation of high frequency underwater acoustic waves. Quantifying the impact of sea surface roughness on the acoustic wave propagation is an important step in both determining performance levels of underwater acoustic instrumentation and developing techniques for using acoustic waves to measure sea surface roughness. This study involves a combined approach based on experimental observation and modeling of both surface waves and acoustic waves in order to assess the detail of acoustic signal interaction with the sea surface.

A high frequency acoustics experiment was conducted during September 22 through September 29, 1997 (HFA97 experiment) in a shallow water region of the Delaware Bay [1]. During the experiment, acoustic signals were transmitted between source-receiver tripods deployed on the sea floor, while highly calibrated environmental data was collected simultaneously from a nearby oceanographic observation platform [2]. Source-receiver tripods were carefully spaced in range so rays with a single surface interaction were easily distinguished in received signals. Extensive analysis of the single surface reflected portion of received signals shows correlation between signal fluctuations and wind speed [1].

In order to further understand the interaction of acoustic waves with the rough airsea boundary, a combined acoustic-ocean surface model has been employed to simulate the time-angle fluctuations observed in shallow water acoustic transmissions. The model combines the BELLHOP ray-based acoustic model [3] and an empirical wind driven sea surface model [4]. The HFA97 data set is used to guide model development and validate results. 


\section{EXPERIMENTAL DATA}

The HFA97 experiment was conducted in a central region of the Delaware Bay at $75^{\circ} 11^{\prime}$ 'West and $39^{\circ} 01^{\prime}$ North. Two bottom mounted tripods, each having an acoustic source and three receiving hydrophones, were placed in $15 \mathrm{~m}$ of water and separated by $387 \mathrm{~m}$. On each tripod, the source was located $3.125 \mathrm{~m}$ above the sea floor and the three receiving hydrophones were located at $0.33,1.33$, and $2.18 \mathrm{~m}$ respectively (Fig. 1). Sources transmitted broad-band chirp signals over the frequency range of $0.6-18.0 \mathrm{kHz}$.

During the experiment, different pulse transmission rates were used so as to capture the fast and slow temporal variations of the acoustic field driven by different physical ocean processes. In one case, the broad-band chirp signal was transmitted every 0.345 $\mathrm{s}$ for a 40 -s interval and then repeated every hour for the entire experiment. During these 40 -s intervals, each received signal had sufficient time to clear before the next signal arrived so that overlapping did not occur.

Analysis presented here focuses on received signals that result from acoustic waves traveling from the source on one tripod to the three remotely mounted hydrophone receivers, located 387 meters away on the opposite tripod. For these signals, the HFA97 experimental design allowed for examination of the time evolution of ray paths involving only one surface interaction [paths 2-5 in Fig. 1 (a)].

In previous HFA97 analysis, remotely received signals across the three hydrophones were used with a beamforming technique to calculate signal arrival angle as a function of arrival time [1]. By considering the geometry of the HFA97 experimental setup [Fig. 1 (a)], the resulting beamformed plots can be used to easily distinguish the portion of the received signal corresponding to Single Surface Reflected (SSR) ray paths. Also, at lower wind speeds, beamformed plots can be used to distinguish between four individual SSR ray paths [Fig. 1 (b)].

During HFA97, several oceanographic and meteorological measurements were made coincident with acoustic measurements which included, tide height, current profiles, sound speed profiles, air temperature, wind speed, and wind direction.

(a)

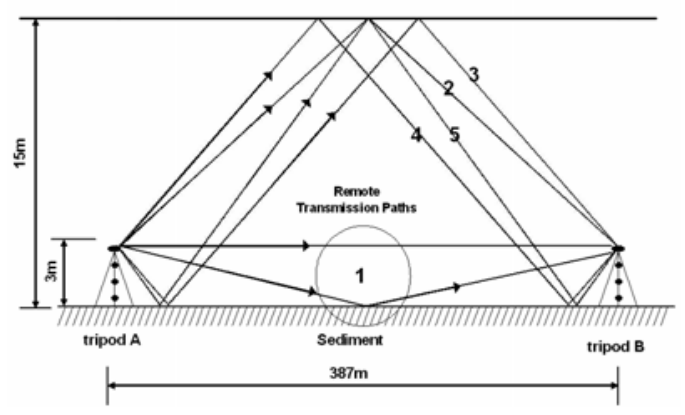

(b)

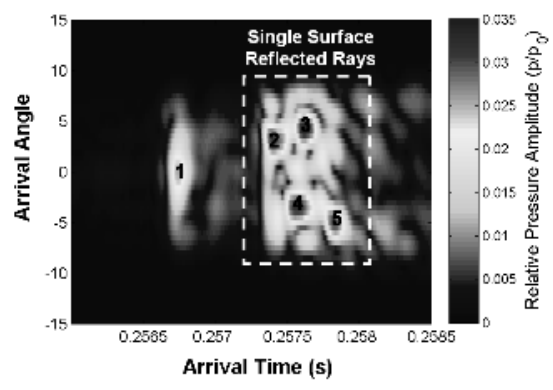

FIGURE 1. (a) HFA97 Experimental setup and ray paths associated with remote transmissions. Single surface reflected ray paths are individually numbered. (b) Remotely received signal arrival angle versus arrival time for a calm period (wind speed of about $2 \mathrm{~m} / \mathrm{s}$ ); single surface reflected ray paths are easily distinguished in the signal [numbers correspond to rays labeled Fig 1(a)]. 


\section{MODELING METHODS}

\section{Ray Theory and Gaussian Beam Tracing}

There have been a number of efforts to modify conventional ray theory in order to develop improved methods that provide more accurate results but retain computational efficiency. One such method is Gaussian beam tracing [3]. With this technique, a fan of rays is traced from a point source with trajectories governed by the standard ray equations. The Gaussian beam method associates with each ray a beam with a Gaussian intensity profile normal to the ray. An additional set of equations which govern beam width and curvature are integrated along with the standard ray equations.

The Gaussian beam tracing method has been adapted to the typical ocean acoustics waveguide and has been implemented as a tool called BELLHOP. This model has rigorously been tested and results show excellent agreement with certain full wave models at high frequencies. The method is free of numerical artifacts affecting standard ray models and still retains the computational efficiency of a ray based approach. As the detail of this model is provided in [3], here we refrain from further explanation.

\section{Modeling the Ocean Surface using JONSWAP}

In coastal regions, the wind acts on a limited fetch. As a result, the sea will not become fully developed and the large-scale or swell components of the waves will be significantly reduced in amplitude. The JONSAWP spectral model computes a sea surface frequency spectrum, $S(\omega)$, under fetch-limited conditions as function of wind speed [4]. This model is based on an extensive wave measurement program (Joint North Sea Wave Project) carried out in 1968 and 1969 in the North Sea. The JONSWAP spectrum provides a good starting point for modeling surface conditions in the area where the HFA experiments were conducted.

The JONSWAP spectral model takes the form:

$$
S(\omega)=\alpha g^{2} \omega^{-5} \exp \left[-\frac{5}{4}\left(\frac{\omega}{\omega_{p}}\right)^{-4}\right] \gamma^{\delta},
$$

where $\delta$ is a peak enhancement factor:

$$
\delta=\exp \left[-\frac{\left(\omega-\omega_{p}\right)^{2}}{2 \sigma_{0}^{2} \omega_{p}^{2}}\right] .
$$

The parameters $\gamma$ and $\sigma_{0}$ are given as $\gamma=3.3, \sigma_{0}=0.07$ for $\omega \leq \omega_{p}$, and $\sigma_{0}=0.09$ for $\omega>\omega_{p}$, while $\alpha$ is a function of fetch, $\mathrm{X}$ and wind speed, $\mathrm{U}$ :

$$
\alpha=0.076\left(\frac{g X}{U}\right)^{-0.22},
$$


and peak frequency $\omega_{p}$ is given as:

$$
\omega_{p}=7 \pi\left(\frac{g}{U}\right)\left(\frac{g X}{U^{2}}\right)^{-0.33} .
$$

A sea surface height wavenumber spectrum, $W(k)$ can be obtained from the JONSWAP frequency spectrum using the relationship $S(\omega) d \omega=W(k) d k$, and the gravity wave dispersion relation, $\omega=\sqrt{k g}$, where $k$ is the wavenumber of ocean waves.

The spectral method can be used to generate one dimensional, sea surface realizations consistent with the JOWNSWAP spectrum [5,6]. Surface heights are generated at $\mathrm{N}$ points with spacing $\Delta x$ across the horizontal range of length $L=N \Delta x$. Realizations with the desired spectral properties can be generated at points $x_{n}=n \Delta x(\mathrm{n}$ $=1, \ldots, \mathrm{N})$ with the following expression for surface height function $f(x)$ :

$$
f\left(x_{n}\right)=\frac{1}{L} \sum_{j=-N / 2}^{N / 2-1} F\left(K_{j}\right) e^{i K_{j} x_{n}}
$$

where for $j>0$,

$$
F\left(K_{j}\right)=\left[2 \pi L W\left(K_{j}\right)\right]^{1 / 2} u
$$

and for $j<0, F\left(K_{j}\right)=F\left(K_{j}\right)^{*}$. In this expression, $K_{j}=2 \pi j / L, u$ indicates an independent sample taken from a zero mean, unit variance Gaussian distribution, and $W(K)$ represents the JONSWAP wavenumber spectrum.

When generating these 1-D surface realizations, surface partition width, $\Delta x$, must be selected. For this modeling study, the dominant wavelength predicted by the JONSWAP spectrum at each wind speed will be used to set $\Delta x$.

When calculating the JONSWAP frequency spectrum for a chosen fetch and wind speed, the model gives a predicted peak frequency of the spectrum, $\omega_{p}$ (4). At each different wind speed, $\omega_{p}$ can be used to calculate a peak wavelength, $\lambda_{p}$ using the deep water dispersion relation and the relationship between wavenumber, $k$ and wavelength, $\lambda$ (where $\lambda=2 \pi / k$ ). Here, $\lambda_{p}$ represents the dominant wavelength of ocean surface waves for the given conditions. For this modeling case, at each wind speed, $\Delta x$ will be set to one half of this dominant wavelength. Surface heights between generated points will be linearly interpolated.

When using the JONSWAP wavenumber spectrum to generate 1-D surface realizations, the total wave energy in the spectrum is applied to waves propagating along the x-axis. This may exaggerate surface roughness slightly. In addition, in this process the out of plane scattering of the acoustic field may be neglected. A better approach would be to use 1-D cross sections through 2-D surface realizations in which the wave energy is also distributed in azimuth. However, the focus of this study is to demonstrate the feasibility of the combined acoustic and surface wave modeling approach only. 


\section{Integration of BELLHOP and Surface Model}

Empirical sea surface models have been combined with acoustic models in past studies of similar nature [6-10]. The modeling approach presented here however is unique in terms of computational efficiency. The concept behind this combined sea surface/acoustic model is the utilization of rough ocean surface realizations and the Gaussian beam tracing model (i.e. BELLHOP [3]). Rough surface realizations are generated using HFA97 wind speed measurements, the JONSWAP wavenumber spectrum, and the spectral method. These surfaces are read into BELLHOP as (horizontal range, surface height) points and become the upper boundary over the water column through which beams are traced. When a beam interacts with the rough surface boundary, the beam trajectory is geometrically reflected from the rough surface, using the beam's angle of incidence and the surface slope at the point of intersection. The resulting model output simulates the fluctuations in arrival angle and arrival time observed in the HFA97 transmissions.

In acoustic wave scattering theory, the scale of ocean surface roughness is usually specified by the surface roughness (Rayleigh) parameter [11] which is defined by, $\chi \equiv 2 k h_{r m s} \sin \left(\theta_{g}\right)$, where $k$ is the acoustic wavenumber, $h_{r m s}$ is the rms sea surface displacement mean level, and $\theta_{g}$ is the grazing angle. For the HFA97 case, using the center frequency of the signal $(12 \mathrm{kHz})$ and the typical $h_{r m s}$ for the region considered (0.2-0.4 m), $\chi \approx 2$ which indicates that the SSR portion of received signals consist of incoherent scattering. This combination of high frequency and large scale roughness justifies the approach of geometrically reflecting acoustic ray paths from individual points on the rough ocean surface.

\section{MODEL RESULTS}

\section{Acoustic Time-Angle Fluctuations}

Time-angle fluctuations of SSR arrivals were measured in the HFA97 data. Timeangle standard deviations were calculated for each hourly, 40-s transmissions consisting of 115 chirp signals.

Beamformed plots [Fig. 1 (a)] can be used to pick out the portion of a received signal that corresponds to a specific ray path. Figure 1 (b) represents a signal that was transmitted during a calm period (wind $<3 \mathrm{~m} / \mathrm{s}$ ) and four individual SSR ray paths can be clearly distinguished. In similar plots for rougher periods, it becomes difficult to distinguish between four individual SSR rays due to the breakup and formation of micro-multi paths resulting incoherent scattering at the rough sea surface. For most rough and calm periods, however, it is feasible to pick out the very first arriving SSR ray path in the second group of arrivals.

Beamformed results were used to track time-angle fluctuations of first SSR arrivals in HFA97 data. Time-angle standard deviations of first SSR arrivals are calculated for the group of signals received during each hourly 40 -s transmission interval. Time- 
angle standard deviations are then plotted against the wind speed recorded at that transmission time.

The BELLHOP/JONSWAP model was used with a Monte Carlo simulation to calculate the standard deviation of arrival time and arrival angle of the first arriving beam with a single surface interaction and no bottom interaction (first SSR beam shown as path 2 in Fig. 1). Separate model runs were made for each one meter/second increment in wind speed (for the range of $1-15 \mathrm{~m} / \mathrm{s}$ ). For each run, 200 surfaces were generated for the given wind speed. A separate BELLHOP beam trace was performed for each of the 200 rough surfaces. Standard deviations of arrival time and arrival angle of the first SSR beams were calculated for each wind speed increment using output from the 200 runs. These standard deviations provide a description of received signal fluctuations which increase with wind speed and surface roughness.

Figure 2 shows comparisons of modeled and measured time-angle standard deviations of the first SSR arrivals. Model results and data agree well for wind speeds of about $9 \mathrm{~m} / \mathrm{s}$ and less. At lower wind speeds, both time and angle standard deviations show an approximately linear increase with wind speed. Model deviation from HFA97 data at higher wind speeds is a possible indication that increased breaking wave activity occurred at the sea surface at higher wind speeds. The sea surface generator used by this model does not consider the nonlinear hydrodynamics of breaking waves. Therefore, at this point, the combined BELLHOP/JONSWAP model is useful for predicting acoustic signal fluctuations at lower wind speeds.
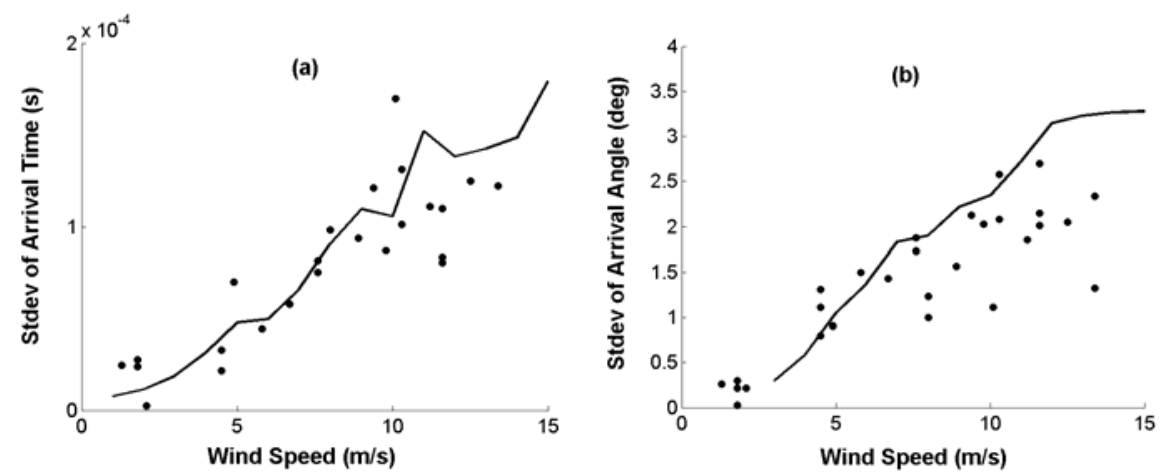

FIGURE 2. Comparisons of BELLHOP/JONSWAP model results obtained from Monte Carlo procedure (solid line) and measured HFA97; fluctuations of single surface bounce beam versus wind speed standard deviation of (a) arrival time in seconds and (b) arrival angle in degrees.

\section{Observed Amplitude Fluctuations}

Modeling signal amplitude fluctuations remains to be explored in subsequent work, as open area of research due to complexities stemming from combined sea surface roughness and interactions between acoustic waves and bubbles resulting from breaking waves. Here, observed signal amplitude fluctuations are presented. Remarkably, these amplitude fluctuations show the same trends as the time-angle fluctuations presented above.

As stated earlier, HFA97 experimental setup was designed so that the portion of remotely received signals corresponding to single surface reflected (SSR) rays is easy 
to distinguish. A method was developed to separate this portion of a received signal in order to calculate mean amplitude across the duration of a SSR portion's arrival time. This average SSR amplitude was calculated for each ping in a 40-s transmission, and then the standard deviation of the group of values was calculated for different wind speeds.

Figure 3 (a) shows a plot of SSR amplitude standard deviation versus wind speed. Similar to the results shown in the time-angle plots above, amplitude fluctuations increase roughly linearly with wind speed and the trend stops after about $9 \mathrm{~m} / \mathrm{s}$ for this data. Figure 3(b) shows the average SSR amplitude calculated for the whole group of 115 pings at each transmission time. This average SSR amplitude remains close to a single value at lower wind speeds and then suddenly drops off at higher wind speeds. This type of decrease in amplitude of surface reflected acoustic waves typically occurs when there are a significant amount of bubbles in the water column near the sea surface [12]. The trends shown in Fig. 3 (a) and (b) provide another possible indication that an increase in breaking wave activity occurred at the ocean surface during periods of higher wind speeds.
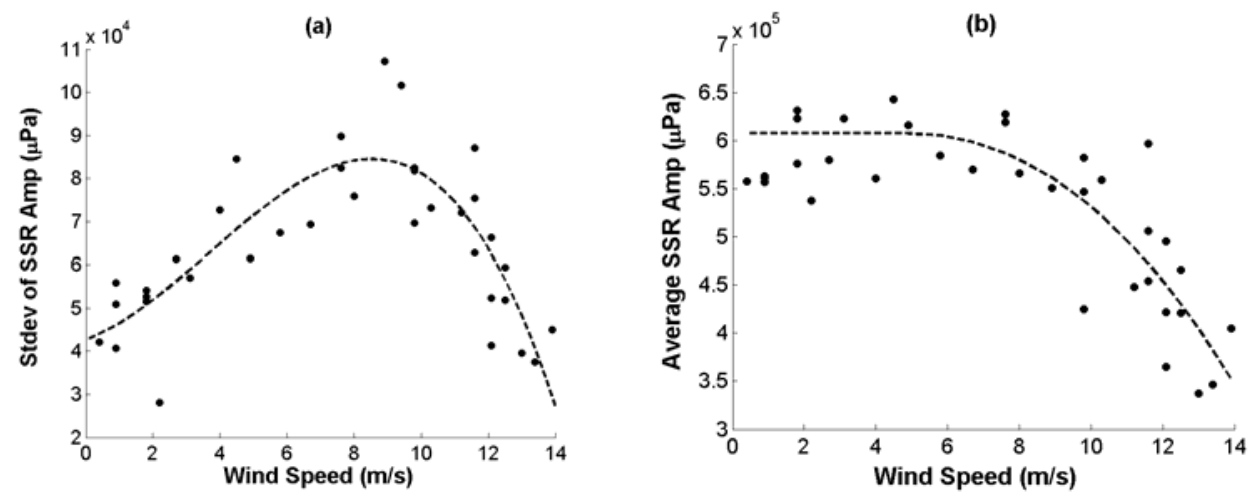

FIGURE 3. HFA97 SSR amplitude fluctuations versus wind speed; (a) measured standard deviation of SSR amplitude for 40-s group of signals (dots) and least squares polynomial fit (line); (b) measured average SSR amplitude 40-s group of signals (dots) and least squares polynomial fit (line).

\section{CONCLUSIONS}

Combining an empirical wind driven sea surface model and a ray-based acoustic model presents a unique approach to predicting fluctuations in acoustic signals induced by sea surface roughness. Tracing beams through sea surface height deviations and changing beam direction at surface reflection based on surface slope results in a realistic simulation of time-angle fluctuations in received signal at lower wind speeds. Also, using ray-based acoustic methods makes the model extremely computationally efficient since multiple model runs can be made quickly supporting timely model modification and improvement.

Initial comparisons between this combined model output and HFA97 observations yield good results for lower wind speeds. Data from other high frequency shallow 
water acoustic experiments will be compared with the model for further validation of this approach. Also, subsequent work will focus on using this modeling approach to predict amplitude fluctuations of acoustic signals induced by fetch limited sea surface roughness.

\section{ACKNOWLEDGMENTS}

The authors wish to thank all participants of the HFA97 experiment, particularly Steve Forsythe for his help in signal processing. Special thanks is due to Michael Porter for providing help with the BELLHOP model. This work was supported by the Office of Naval Research, code 321OA and in part by the Sea Grant program.

\section{REFERENCES}

1. Badiey, M., Mu, Y., Simmen, J.A., Forsythe, S.E., "Signal Variability in Shallow-Water Sound Channels," IEEE Ocean Eng. 25 (4), 2000, pp. 492-500.

2. Badiey, M., Lenain, L. Wong, K.C., Heitsenrether, R., Sundberg, A., "Long-term Acoustic Monitoring of Environmental Parameters in Estuaries," in Proc.Oceans 2003 Marine Technology and Ocean Science Conference, San Diego, CA.

3. Porter, M.B., Bucker, H.P., "Gaussian Beam Tracing for Computing Ocean Acoustic Fields, "J. Acoust. Soc. Am. 82 (4), 1987, pp. 1348-1359.

4. Hasselmann, D., Dunckel, M., and Ewing, J.A., "Directional Wave Spectra Observed During JONSWAP 1973," Jour. Phys. Ocean. 10, 1980, pp. 1264-1280.

5. Ogilvy, J.A., Theory of Wave Scattering from Random Rough Surfaces (Institute of Physics Publishing, Bristol and Philadelphia, 1991) pp. 228-229.

6. Thorsos, E.I., “Acoustic scattering from a 'Pierson-Moskowitz' sea surface”, J. Acoust. Soc. Am. 88 (1), 1990, pp. 335-349.

7. McDaniel, S.T., "Composite-Roughness Theory Applied to Scattering From Fetch Limited Seas, "J. Acoust. Soc. Am. 82 (5), 1987, pp. 1712-1719.

8. Dahl, P.H., "On the Spatial Coherence and Angular Spreading of Sound Forward Scattered From the Sea Surface: Measurements and interpretive model," J. Acoust. Soc. Am. 100(2), 1996, pp. 748-758.

9. Dahl, P.H., "On Bistatic Sea Surface Scattering: Field Measurements and Modeling," J. Acoust. Soc. Am. 105 (4), 1999, pp. 2155-2169.

10.Dahl, P.H. "High-Frequency Forward Scattering from the Sea Surface: The Characteristic Scales of Time and Angle Spreading," IEEE Ocean Eng. 26, 2001, 141-151.

11. Clay, C.S., Medwin, H. (1977). Acoustical Oceanography (Wiley-Interscience Publications), $49-51$.

12. Ostrovsky, L.A., Sutin, M.S., Soustova, I.A., Matveyev, A.L., Potapov, A.I., Kluzek, Z. "Nonlinear scattering of acoustic waves by natural and artificially generated subsurface bubble layers in the sea," J. Acoust. Am. 113, 2003, pp. 741-749. 